\title{
Influence of Water Quality on Cholesterol-Induced Tau Pathology: Preliminary Data
}

\author{
D. Larry Sparks, ${ }^{1}$ Chuck Ziolkowski, ${ }^{1}$ Todd Lawmaster, ${ }^{2}$ and Timothy Martin ${ }^{2}$ \\ ${ }^{1}$ Roberts Laboratory for Neurodegenerative Disease Research, Banner Sun Health Research Institute, Sun City, AZ 85351, USA \\ ${ }^{2}$ Animal Care Program, St. Joseph's Hospital and Medical Center, 350 W. Thomas Road., Phoenix, AZ 85013, USA
}

Correspondence should be addressed to D. Larry Sparks, larry.sparks@bannerhealth.com

Received 30 December 2010; Accepted 12 February 2011

Academic Editor: Rosanna Squitti

Copyright (c) 2011 D. Larry Sparks et al. This is an open access article distributed under the Creative Commons Attribution License, which permits unrestricted use, distribution, and reproduction in any medium, provided the original work is properly cited.

\begin{abstract}
The studies employed the cholesterol-fed rabbit model of Alzheimer's disease (AD) to investigate the relationship between AD-like neurofibrillary tangle (NFT) neuropathology and tau protein levels as the main component of NFT. We measured brain and plasma tau levels and semiquantified NFT-like neuropathology in cholesterol-fed rabbits administered drinking water of varying quality (distilled, tap, and distilled+copper) compared to animals receiving normal chow and local tap water. Total tau levels in plasma were increased in all cholesterol-fed rabbits compared to animals on normal chow, regardless of quality of water. In contrast, increased tau in brain and increased AT8 immunoreactive NFT-like lesions were greatest in cholesterol-fed rabbits administered distilled water. A substantial decrease in brain tau and incidence and density of AT8 immunoreactive NFT-like lesions occurred in cholesterol-fed rabbits administered copper water, and an even greater decrease was observed in cholesterol-fed animals on local tap water. These studies suggest the possibility that circulating tau could be the source of the tau accumulating in the brain.
\end{abstract}

\section{Introduction}

One of the neuropathologic features invariably found in the brains of individuals with Alzheimer's disease (AD) is the amyloid-beta $(\mathrm{A} \beta)$ containing senile plaque $(\mathrm{SP})$. Blood samples have been assessed for changes in $\mathrm{A} \beta$ levels-driven by the abundant data that $A \beta$ in the brain was the prime candidate for precipitating $\mathrm{AD}$. We showed as did others that circulating levels of the shorter form of $\mathrm{A} \beta(\mathrm{A} \beta 40)$ increased with decreasing cognitive performance within a control population. Levels of $A \beta 40$ were increased even further in mild cognitive impairment (MCI) compared to controls, and circulating levels of $\mathrm{A} \beta 40$ are decreased in $\mathrm{AD}$ compared to MCI [1]. We suggested that gradually increased production of $A \beta$ occurred with effective clearance from the brain when individuals change from control to MCI and that reduced clearance was evidenced as lower circulating $\mathrm{A} \beta$ levels with concurrently increased central accumulation in the transition from MCI to $\mathrm{AD}$ [1].

This is consistent with findings in the cholesterol-fed rabbit model of human coronary artery disease where we identified numerous neuropathologic features of $\mathrm{AD}$ including central accumulation of $\mathrm{A} \beta$. Early studies were performed in animals administered a diet containing $2 \%$ cholesterol and tap water for only 8 weeks compared to animals administered normal rabbit chow and tap water for a similar length of time [2]. Further studies suggested that the induction of $\mathrm{AD}$-like $\mathrm{A} \beta$ neuropathology by dietary cholesterol depended on the quality of water the rabbit was drinking. Animals fed $2 \%$ cholesterol and drinking distilled water showed minimal AD-like neuropathology, whereas animals drinking tap water were severely affected [3]. Subsequent studies indicated that it was the copper in the tap water that produced the difference in severity of the cholesterol-produced $\mathrm{AD}$-like $\mathrm{A} \beta$ pathology [4]. It was clear that cholesterol caused the overproduction of $A \beta$ in the brain and copper influenced its clearance to the blood via inhibition of LRP at the vascular interface $[5,6]$.

Copper has been implicated in the progression and possibly the cause of AD. Morris et al. have shown that increased copper intake significantly increases the rate of progression of $\mathrm{AD}$ in the setting of elevated fat intake [7]. A role for 
altered copper metabolism as a cause of neurodegenerative disorders other than $\mathrm{AD}$ is clearly recognized [8], and a role for copper in $\mathrm{AD}$ is emerging. Ceruloplasmin transports $85-95 \%$ of the copper in human blood [8]. Both copper and ceruloplasmin levels have been shown to be elevated in the blood of patients with $\mathrm{AD}$ compared to controls by most [9-13], but not all, investigators [14]. Squitti et al. have reported a significant increase in circulating copper in $\mathrm{AD}$ and a trend for increased ceruloplasmin [15]. These authors also reported a significant negative correlation in $\mathrm{AD}$ between increased copper/ceruloplasmin and decreased scores on the MMSE, AVLT-A7, and the clock draw [15, 16]. Similar to Squitti's data, we have shown that there are significant increases in blood copper/ceruloplasmin in $\mathrm{AD}$ compared to age-matched controls [1]. Moreover, circulating copper/ceruloplasmin levels increased in controls with lower performance on the AVLT-A7 and MMSE and remained elevated in $\mathrm{MCI}$ and $\mathrm{AD}$ as cognitive ability progressively deteriorated [1].

Although found in many other tissues of the body [17], tau protein in brain is the precursor for the other important neuropathologic feature of $\mathrm{AD}$, the neurofibrillary tangle (NFT). Specifically phosphorylated-tau (p-tau) is a main component of the NFT [18]. Single strands of p-tau become paired into filaments called paired helical filaments (PHF), which then assemble into fibrillary complexes within a neuron eventually becoming an NFT. It has been reported that there are increased tau and 181-p-tau levels in MCI subjects converting to $\mathrm{AD}$ and that levels are stable in controls and MCI subjects not converting to AD [19]. Although there are reports that the protein tau may have a copper binding site [20-22], there is only one study investigating any relationship between the protein tau and copper in the blood and CSF in AD [16], where a significant positive relationship $(P<.03)$ between copper levels in the serum and tau levels in the CSF was identified in $\mathrm{AD}$ compared to controls. Nevertheless, there are no reports of changing cognitive performance (i.e., $\mathrm{AD}$ versus control) and a relationship between CSF copper and tau levels or copper and tau levels in the circulation.

We recently measured total tau in human plasma and have found significant differences between cognitively normal individuals, subjects with $\mathrm{MCI}$, and patients with $\mathrm{AD}$ (unpublished observation). Tau levels (total human tau) were established in each plasma sample using ELISA, kits and standards purchased from Invitrogen Corp., Camarillo, CA. The mean circulating tau level (picograms $/ \mathrm{mL}$ ) is depressed in plasma of patients with $\mathrm{AD}$ when compared to both cognitively normal control and individuals with mild cognitive impairment (MCI) $(P<.0001$ and .0002 , resp. $)$. The mean tau levels were also significantly reduced in MCI compared to control $(P=.048)$, and a significant age-related increase was identified $(P=.042)$ in the control population (unpublished observation).

In our previous rabbit studies we administered $2 \%$ cholesterol and varying qualities of drinking water, and we were able to manipulate $\mathrm{A} \beta$ accumulation and memory but were unable to identify any changes in tau as the major component of the other characteristic lesion in AD-NFT. A recent publication indicated that administering lower levels of dietary cholesterol (1\%) over longer periods of time (7 months) could produce NFT-like changes in the brain along with $\mathrm{A} \beta$ deposition in female rabbits [23]. An even more recent publication found similar features and behavioral dysfunction in a hypercholesterolemic rat model [24]. Using the same methods employed in the current study, the authors note increased $\mathrm{A} \beta$, tau, and $\mathrm{p}$-tau in the cortex of cholesterolfed rats [24].

Antibodies have been developed to detect different stages of the NFT formation, including PHF-1 antibody identifying PHF and AT8 antibody highlighting p-tau [18]. Seven months of $1 \%$ cholesterol diet caused increased PHF-1 immunoreactivity in hippocampus of female rabbits [23] as well as plaque-like $A \beta$ deposition in the hippocampus and cortical brain regions [25]. We performed a pilot study of female rabbits fed $1 \%$ cholesterol and drinking distilled water, copper containing distilled water, or local tap water compared to animals administered normal chow and tap water. In this preliminary study we focus on changes in tau by measuring total tau levels by ELISA and evaluating the brain for the presence of p-tau by performing AT8 immunohistochemistry.

\section{Methods}

Twenty female New Zealand White (NZW) rabbits approximately 2.5 kilograms body weight were obtained, acclimated to the vivarium for 8-10 days, and assigned to one of four groups. Fifteen of them were fed 1\% Cholesterol Diet purchased from Purina TestDiet, the remaining five were fed normal high-fiber chow from Purina LabDiet. The control animals along with five of the cholesterol fed animals were allowed distilled water to drink. Five of the remaining rabbits received local tap water. and the other five were administered distilled water with copper ion added (0.12 PPM copper ion as sulfate). US Filter's analysis of the local Sun City tap water used can be seen in a previous publication [5]. Late in the study, 2 of 5 animals fed the $1 \%$ cholesterol chow developed fatty liver disease and/or severe atherosclerosis. These animals were anesthetized and perfused with normal saline as soon as symptoms of either disease appeared. Counter-part animals in the other three groups were sacrificed and similarly processed for tissue collection. Samples collected from these animals have not yet been investigated.

Blood samples were collected prior to initiating administration of the experimental diet and water (baseline). At the end of 21 weeks (5 months) animals were sacrificed after each rabbit was deeply anesthetized with Ketamine (50$75 \mathrm{mg} / \mathrm{kg})$ and Xylazine $(5 \mathrm{mg} / \mathrm{kg})$ given intramuscularly in the upper thigh. Additional Ketamine was given at $20 \%$ of the original dose, if it was determined that the animal was not deep enough. Once the rabbit was anesthetized, the chest and cranium were shaved, and the animal was placed on an embalming board. An incision was made over the sternum and ribs are reflected to expose the heart. A 23-gauge vacutainer needle was introduced into the left 
ventricle, and $10 \mathrm{cc}$ of blood is collected in purple top tubes. The needle was removed and replaced with a 23 -gauge 1.5 inch catheter attached to an IV line. The catheter is advanced into the left atrium and secured into place using a vascular clamp. The right atrium is then cut to facilitate drainage using a pair of Mayo scissors. The rabbit was then perfused with a minimum of 120 milliliters of sterile saline using a syringe pump set to deliver $600 \mathrm{cc}$ per hour. Once the animal was perfused, various organs were collected, including the brain, heart, liver, spleen, and kidneys; half of the brain was dissected fresh (frontal pole, whole hippocampus), frozen on dry ice and then stored at $-70^{\circ} \mathrm{C}$, and the other organs and half-brain were emersion-fixed in $4 \%$ paraformaldehyde.

2.1. Tissue Preparation for ELISA Assays. Brain samples were homogenized thoroughly in a cold solution $(1: 8 ; \mathrm{w}: \mathrm{v})$ of $5 \mathrm{M}$ guanidine $\mathrm{HCl}$ and $50 \mathrm{mM}$ Tris $\mathrm{HCl}, \mathrm{pH} 8.0$ using a Dounce homogenizer. This solution was transferred to an Eppendorf tube and mixed at room temperature for 34 hours. This sample was diluted 1:50 with a Dulbecco's phosphate buffered saline with 5\% BSA and $0.03 \%$ Tween20, pH 7.4 supplemented with protease inhibitor cocktail (Sigma P8340 or Calbiochem no. 539131) at $1 \mathrm{mM}$. This diluted sample was centrifuged at $16,000 \mathrm{~g}$ for 20 minutes at $4^{\circ} \mathrm{C}$ prior to use. A fifty $\mu \mathrm{L}$ aliquot of each sample was assessed in the Tau ELISA assay.

Frozen plasma samples were slowly thawed, and a $20 \mu \mathrm{L}$ aliquot was diluted $1: 10$ with undiluted "standard dilution buffer" provided with each Tau ELISA kit. A fifty $\mu \mathrm{L}$ aliquot of this solution was added to the appropriate well in the following assay.

2.2. Tau ELISA Assay. Samples were assayed using the ELISA kit from Invitrogen (\#KHB0042). All reagents used were supplied in the kit including the "standards dilution buffer". Wash buffer was prepared by diluting the supplied concentrate $1: 25$ with purified water. Standards were prepared by diluting the supplied standard with "standard dilution buffer" as specified on labeling to $2000 \mathrm{pg} / \mathrm{mL}$. Serial dilutions were made into standard dilution buffer to 1000,500 , $250,125,62.50$, and $31.25 \mathrm{pg} / \mathrm{mL} .100 \mu \mathrm{L}$ of standards were added to wells in duplicate. $50 \mu \mathrm{L}$ of standard dilution buffer was added to the rest of the wells. $50 \mu \mathrm{L}$ of sample (fresh frozen brain or plasma collected in a purple top vacutainer) was added to ELISA wells in duplicate. The ELISA plate was covered and incubated for 2 hours at room temperature on a rotary shaker $(1200 \mathrm{rpm})$ and thereafter washed 4 times. A $100 \mu \mathrm{L}$ aliquot of anti-tau detection antibody was added to each well, and the plate was incubated as above for 1 hour and thereafter washed 4 times. Antirabbit HRP concentrate was diluted 100x into HRP diluent and added to wells at $100 \mu \mathrm{L} /$ well, and the plate is incubated for 30 minutes. After emptying the plate $100 \mu \mathrm{L}$ of stabilized chromogen was added to each well, and the uncovered plate was incubated at room temperature in the dark for 20-30 minutes on the rotary shaker. The reaction was completed by addition of $100 \mu \mathrm{L}$ of provided "stop" solution, and the plate was then read at $450 \mathrm{~nm}$ in a Bio-Tex ELx800 plate reader. Reader software calculated standard curve, concentration, SD, and CV\% (4 parameter algorithm).

2.3. AT8 and $A \beta$ Immunohistochemistry. Blocks of paraformaldehyde fixed tissue were affixed to a metal block with superglue, and $50 \mu \mathrm{m}$ vibratome sections were collected and stored in $4 \%$ paraformaldehyde until use. On the first day, $50 \mu \mathrm{m}$ thick vibratome tissue sections were washed twice in $0.01 \mathrm{M}$ Tris Buffered Saline (TBS) pH 7.6 for 10 minutes. All steps were carried out on orbital shaker rotating at about $500 \mathrm{rpm}$. Sections were then transferred to a solution of $3 \% \mathrm{H}_{2} \mathrm{O}_{2}$ in $0.01 \mathrm{M}$ TBS and incubated at room temperature for $30 \mathrm{~min}$. Sections were then washed twice for 10 minutes in $0.01 \mathrm{M}$ TBS $+0.1 \%$ triton $\mathrm{X}-100 \mathrm{pH} 7.6$. Sections were transferred to $88 \%$ formic acid for $2-3 \mathrm{~min}$ and washed twice for 10 minutes in $0.01 \mathrm{M}$ TBS $+0.1 \%$ Triton X-100 $\mathrm{pH} 7.6$ and then transferred to antigen retrieval solution (GeneTex, \#GTX28194) for 12-15 minutes. Sections were washed again and then incubated in 3\% horse serum in 0.01 M TBS + 0.1\% Triton X-100 pH 7.6 for 1 hour. Sections were then washed twice for 10 minutes in $0.01 \mathrm{M}$ TBS $+0.1 \%$ triton X-100 pH 7.6 and transferred to $1^{\circ}$ antibody $(1: 500$ dilution of antihuman PHF-Tau AT8 monoclonal antibodyPierce Biotechnology or 1:100 dilution of 10D5 antibody to $\mathrm{A} \beta$-Elan pharmaceuticals) in $0.01 \mathrm{M}$ TBS $+0.1 \%$ triton $\mathrm{X}-100 \mathrm{pH}$ 7.6) overnight at $4^{\circ} \mathrm{C}$. On the following day the sections were washed once in $0.01 \mathrm{M}$ TBS $+0.1 \%$ Triton $\mathrm{X}-100 \mathrm{pH} 7.6$ and transferred to $2^{\circ}$ antibody (Vectastain ABC kit PK-6102 mouse IgG, 1 drop/10 mL 0.01 M TBS + $0.1 \%$ Triton $\mathrm{X}-100+1 \%$ horse serum) for 1 hour. Then the sections were washed once in $0.01 \mathrm{M}$ TBS $+0.1 \%$ Triton X$100 \mathrm{pH} 7.6$ and transferred to A/B solution (Vectastain kit, 4 drops $\mathrm{A}+4$ drops $\mathrm{B} / 10 \mathrm{~mL} 0.01 \mathrm{M}$ TBS $+0.1 \%$ Triton X100), washed twice for 10 minutes in $0.05 \mathrm{M}$ Tris $\mathrm{pH} 7.9$ and then developed in a diaminobenzidine solution in dark ( $15 \mathrm{mg} \mathrm{DAB}+20 \mu \mathrm{L} 30 \% \mathrm{H}_{2} \mathrm{O}_{2}$ in $100 \mathrm{~mL} 0.05 \mathrm{M}$ Tris) for 6-10 minutes. The reaction was stopped by transferring the sections back into $0.05 \mathrm{M}$ Tris $\mathrm{pH}$ 7.9. Sections were then mounted, serially dehydrated, and coverslipped.

2.4. Statistics. Mean tau levels $\pm \mathrm{SD}$ are present. The groups were compared for differences by ANOVA followed by independent $t$-test. Although mean differences were substantial, the limited number of animals assessed in this preliminary study precluded significance greater than trends $(.05<P<.10)$.

\section{Results}

We performed a pilot study of long-term dietary cholesterol in four groups of rabbits. One group was administered normal chow and tap water, and three groups were administered $1 \%$ cholesterol for five months. The cholesterolfed groups were administered either distilled water, distilled water supplemented with 0.12 PPM copper ion, or local tap water (routinely containing 0.20 PPM copper ion as well as other trace elements and compounds). Employing methods 
TABLE 1: Tau levels in plasma, whole hippocampus, and frontal cortex, and incidence and density of NFT-like AT8 immunoreactive neurons in the frontal and temporal cortices in rabbits grouped according to diet and water quality. The four groups of rabbits were female rabbits fed (1) normal rabbit chow and provided local tap water to drink (Normal chow-tap), (2) 1\% cholesterol diet and distilled drinking water (1\% Cholesterol-DW), (3) 1\% cholesterol diet and copper supplemented distilled drinking water (1\% Cholesterol-DW/Cu), or (4) $1 \%$ cholesterol diet and local tap water ( $1 \%$ Cholesterol-tap) for 5 months.

\begin{tabular}{|c|c|c|c|c|}
\hline Group & Normal chow-tap & 1\% Cholesterol-DW & 1\% Cholesterol-DW/Cu & 1\% Cholesterol-tap \\
\hline$N$ & 3 & 3 & 3 & 3 \\
\hline Tau level Plasma & $24.8 \pm 7.7$ & $35.6 \pm 3.7$ & $37.2 \pm 3.1$ & $34.4 \pm 6.8$ \\
\hline Tau level Hippocampus & $40.4 \pm 11.5$ & $216.1 \pm 71.3$ & $148.4 \pm 48.8$ & $71.8 \pm 33.3$ \\
\hline Tau level frontal cortex & $36.9 \pm 15.9$ & $294.2 \pm 130.6$ & $62.9 \pm 1.9$ & $53.1 \pm 32.7$ \\
\hline $\begin{array}{l}\text { Density of AT } 8 \text { neurons in } \\
\text { frontal cortex }\end{array}$ & none & abundant & very few isolated & Single faint \\
\hline $\begin{array}{l}\text { Number of animals with } \\
\text { AT8 stained Neurons in } \\
\text { frontal cortex }\end{array}$ & 0 of 3 & 3 of 3 & 2 of 3 & 1 of 3 \\
\hline $\begin{array}{l}\text { Density of AT8 neurons } \\
\text { Temporal Cortex }\end{array}$ & none & $\begin{array}{l}\text { Abundant patches of } \\
\text { multiple cells }\end{array}$ & numerous isolated & few isolated \\
\hline $\begin{array}{l}\text { Number of Animals with } \\
\text { AT8 neurons in temporal } \\
\text { cortex }\end{array}$ & 0 of 3 & 3 of 3 & 3 of 3 & 2 of 3 \\
\hline
\end{tabular}

to measure total tau in human plasma, we measured total tau levels in plasma, hippocampus, and frontal cortex in each animal sacrificed after 5 months of dietary manipulation. We also assessed the hippocampus and frontal and temporal cortex for AT8 staining of p-tau and the frontal and temporal cortex for $\mathrm{A} \beta$ accumulation (Figure 1, A4-D4). Plasma samples were taken from each animal at baseline and at sacrifice 21 weeks after initiating experimental diet and controlled diet and water. It is important to note that even though previous studies administered a 1\% cholesterol diet and purified water for 7 months $[23,25]$, we decided to leave open the possibility of identifying enhanced tau pathology in animals on copper containing distilled water and/or tap water and administered experimental diet and drinking water for only 5 months.

We initially had five animals in each of the four groups, but an animal on 1\% cholesterol and tap water became ill after 4 months of diet and was sacrificed along with one animal in each of the other groups. After 4.5 months of diet another $1 \%$ cholesterol and tap water animal became ill as well as an animal on 1\% cholesterol and copper supplemented distilled water-these two animals and a counterpart animal in the other 2 groups were sacrificed. Accordingly there were only 3 animals in each group that completed the 5-month pilot study.

Plasma levels of tau were increased by $40-50 \%$ in each of the cholesterol-fed animal groups after 5 months of experimental diet compared to animals on normal rabbit chow and tap water (Table 1). Tau levels in fresh hippocampus were 5 -fold higher in animals administered 1\% cholesterol and distilled water compared to animals on normal rabbit chow and tap water; levels of tau were 3-fold higher in animals on $1 \%$ cholesterol and copper supplemented distilled water and less than 2-fold in animals on 1\% cholesterol and tap water (Table 1). A similar graded effect on AT8 staining of ptau was glaringly apparent in sections of fixed hippocampus
(Figure 1). In the fascia dentata of the hippocampus AT8 staining is increased dramatically in animals on cholesterol and distilled water (Figure 1, B1), is less pronounced in the animals on cholesterol and copper (Figure 1, C1), and is further reduced in animals on cholesterol and tap water (Figure 1, D1), all compared to animals on normal chow and tap water (Figure 1, A1). This is equally apparent when viewing the whole hippocampus at low power (Figure 1, 4X; A2-D2). These data support the concept that increasing tau levels are directly related to the severity and intensity of AT8stained features in the hippocampus. And on the flip side, the data support the premise that our measured levels of tau in plasma and brain tissue are valid and accurate. Further support of this comes from finding similar graded effects when assessing frontal cortex. In the frontal cortex we found a 6 -fold increase of tau in animals on 1\% cholesterol diet and distilled water compared to animals fed normal rabbit chow. In contrast we found a less than 2-fold increase in tau levels in the frontal cortex of cholesterol-fed animals on copper-supplemented distilled water and tap water (Table 1). Identical to the hippocampus, the number and severity of NFT-like lesions deposits identified in the frontal cortex by AT8 staining correlated to the levels of tau measured (data not shown). AT8 immunoreactive neurons were not observed in the frontal cortex of any animal on normal rabbit chow and tap water. In all of the cholesterol-fed rabbits Becomes administered distilled water to drink, there were abundant AT8 stained NFT-like lesions in frontal cortex (Table 1). The frontal cortex in two of three cholesterolfed rabbits drinking copper supplemented distilled water exhibited a few AT8 stained cells showing fine processes and considerable arborization (less NFT-like; Table 1). Only one animal fed cholesterol diet and drinking tap water showed a single faintly stained AT8 immunoreactive neuron in the frontal cortex. In the superior temporal cortex no AT8 immunoreactive neurons were observed in animals fed 


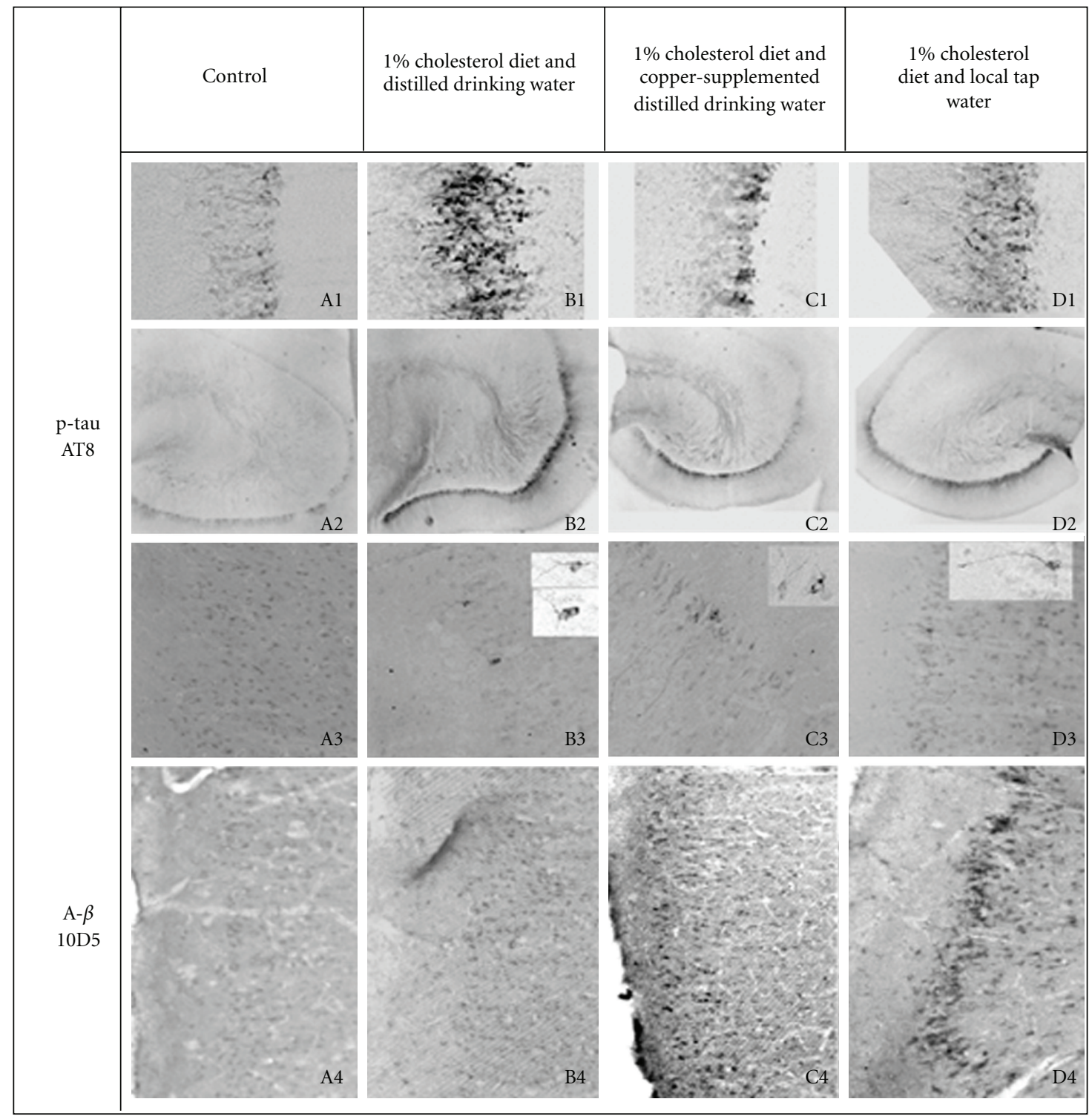

FIGURE 1: Water quality induced differences in immunohistochemical staining for phosphorylated tau (p-tau, AT8) and amyloid-beta (A $\beta$; 10D5) in the brains of cholesterol-fed rabbits compared to animals fed normal control diet. The four groups of rabbits were comprised of female rabbits fed normal rabbit chow and provided local tap water to drink (A1-A4), 1\% cholesterol diet and distilled drinking water (B1B4), $1 \%$ cholesterol diet and copper supplemented distilled drinking water (C1-C4), or 1\% cholesterol diet and local tap water (D1-D4) for 5 months. Representative p-tau AT8 staining in fascia dentate (A1-D1, 10X), hippocampus (A2-D2, 4X), and superior temporal cortex (A3-D3, 10X-insets 40X), and A $\beta 10 \mathrm{D} 5$ staining in superior temporal cortex (A4-D4, 10X) are shown.

normal chow and drinking tap water (Table 1; Figure 1, A3), but 3 of 3 animals fed 1\% cholesterol diet and distilled water demonstrated abundant AT8 immunoreactive features which occurred in patches of 2-3 NFT-like lesions (Table 1; Figure 1, B3). All three of the cholesterol-fed animals drinking distilled water supplemented with copper showed numerous isolated AT8 stained NFT-like lesions in the superior temporal cortex (Table 1; Figure 1, C3). Two of three animals fed cholesterol drinking tap water showed very few isolated cells stained with AT8 antibody (Table 1, Figure 1, D3).

Adjacent sections of hippocampus, and frontal and temporal cortex were stained for the presence of $\mathrm{A} \beta$ using
10D5 antibody employing the detailed methods. As expected, we found that administration of copper containing distilled water or tap water to drink increased the accumulation of $\mathrm{A} \beta$ in hippocampus (not shown) and frontal cortex (not shown) and superior temporal cortex (Figure 1, A4-D4) of animals fed $1 \%$ cholesterol diet compared to animals drinking unaltered distilled water, as found previously in rabbit fed 2\% cholesterol diet. Similar to findings in animals fed $2 \%$ cholesterol diet, we observed a graded effect on $\mathrm{A} \beta$ accumulation in animals fed 1\% cholesterol diet, with the greatest $\mathrm{A} \beta$ accumulation occurring in animals administered tap water (Figure 1, D4), somewhat less accumulation in 
animals administered copper supplemented distilled water to drink (Figure 1, C4), while there is minimal $\mathrm{A} \beta$ staining in the superior temporal cortex of animals drinking distilled water (Figure 1, B4), all compared to animals administered normal chow and drinking tap water (Figure 1, A4). Overall, there seemed to be an inverse relationship between neuronal accumulation of $\mathrm{A} \beta$ and increased levels of tau and associated AT8 immunoreactive NFT-like lesions, based on varying water quality and region of brain. This disconnect suggests that accumulation of $\mathrm{A} \beta$ may not cause increased levels of tau, and that once sufficient elevations tau occur in the brain, this in turn leads to deposition of p-tau containing NFT-like lesions.

\section{Discussion}

This is the first study of the effect of intake of copper or trace metals on levels of the protein tau in the blood and brain. We have shown that there is increased tau neuropathology associated with production of increased tau levels in the blood and brain of the cholesterol fed rabbit. This could be similar to induction of accelerated aging as these findings are consistent with our unpublished observation of age-related increases in plasma tau levels in cognitively normal humans coupled with reported increases in the occurrence and severity of NFT pathology with increasing age in the absence of dementia [26, 27]. The inverse relationship between tau and $\mathrm{A} \beta$ pathology depending on water quality is difficult to reconcile when attempting to apply conventional wisdom, where the accumulation of $\mathrm{A} \beta$ containing SP is thought to precede the occurrence of p-tau containing NFT in AD and that the processes leading to the formation of the lesions may be linked. What we may have uncovered is that these processes may actually not be linked at all. In this cholesterolfed rabbit model of $\mathrm{A} \beta$ and tau neuropathology, we show that increased accumulation of $\mathrm{A} \beta$ occurs concurrently with reduced levels of tau and the incidence of AT8 stained NFT-like lesions. It must be remembered that plaque-only and NFT-only variants of AD have been reported [28, 29]. Neuropathologic evaluation revealed that most clinically diagnosed $\mathrm{AD}$ patients had both plaques and tangles, although in the rare circumstance there were individuals who had only senile plaques or only NFT in their brains $[28,29]$. Coming full circle there are those who would contend that these individuals were misdiagnosed and those demented patients with only plaques or tangles did not have $\mathrm{AD}$ at all (William R. Markesbery, personal communication). This debate continues.

An important feature of animal research is the opportunity to disclose possible mechanisms to explain observations in human studies. Much of disclosing a likely mechanism for altered levels of total tau in plasma in relation to tau concentration in the brain would depend on the origin of the peptide deposited in the brain. If, as all evidence suggests, $\mathrm{A} \beta$ accumulates in the brain with increasing change in the vasculature, which in turn leads to reduced clearance of the toxin to the blood $[5,6]$ and eventually the liver [30], it is difficult to envision how such an alteration in the vascular blood brain barrier (BBB) leads to increased levels of tau and associated formation of NFT-like lesions in the brain-unless the tau accumulating in brain has its origin in the blood. Assuming that 1\% cholesterol diet increases tau plasma levels by $40-50 \%$ in each cholesterol-fed animal groups, the question is how this similar increase in blood tau has differential effects on tau accumulation in the brain, in terms of tau levels and AT8 immunoreactive NFT-like lesions (5-6 fold increases in the "distilled water" group versus 2-3 fold increases in the "distilled water + copper" group, versus $40 \%$ in the "tap water" group)? In other words, the evidence presented would suggest that reduced BBB permeability induced by varying water quality attenuates brain uptake of tau from the blood at the same time it reduces the clearance of $\mathrm{A} \beta$. Therefore, a normally functioning $\mathrm{BBB}$ in animals administered cholesterol and distilled water allows clearance of $\mathrm{A} \beta$ concomitant with central accumulation of tau due to a concomitant increase in the concentration of tau in the blood. By the addition of copper to the distilled drinking water there is a decrease in BBB permeability leading to either an increased accumulation of $\mathrm{A} \beta$ or a decrease in tau levels and associated AT8 immunoreactive features in the brain, while tau concentrations remain elevated to the same level in general circulation. By further reducing water quality by administration of tap water there is even greater compromise in $\mathrm{BBB}$ permeability and an even greater accumulation of $\mathrm{A} \beta$ and a greater reduction of tau levels and AT8 immunoreactive NFT-like lesions in the brain, again while circulating concentrations of tau remain elevated to the same level. Accordingly it can be proposed that tau deposited in the brain of the cholesterol-fed rabbit model of $\mathrm{AD}$ may have come from the circulation. However, it remains unclear how a neuron might take up extracellular tau protein for NFT formation.

Although provocative, one must keep in mind that these are preliminary data and as such require replication. Even so it may be all well and good to be able to induce the production of NFT-like lesions in the brains of cholesterolfed rabbits, but our eventual goal would be much more important-to find a method or medication to make them go away or not form in the first place.

\section{Acknowledgments}

This research was funded in part by the Arizona Biomedical Research Commission (DLS; contract 08-09) the Banner SHRI Arizona Alzheimer's Research Center (AGR-2009017).

\section{References}

[1] D. L. Sparks, S. Petanceska, M. Sabbagh et al., "Cholesterol, copper and $\mathrm{A} \beta$ in controls, $\mathrm{MCI}, \mathrm{AD}$ and the $\mathrm{AD}$ cholesterol-lowering treatment trial (ADCLT)," Current Alzheimer Research, vol. 2, no. 5, pp. 527-539, 2005.

[2] D. L. Sparks, "Intraneuronal $\beta$-amyloid immunoreactivity in the CNS," Neurobiology of Aging, vol. 17, no. 2, pp. 291-299, 1996. 
[3] D. L. Sparks, J. Lochhead, D. Horstman, T. Wagoner, and T. Martin, "Water quality has a pronounced effect on cholesterolinduced accumulation of Alzheimer amyloid $\beta(\mathrm{A} \beta)$ in rabbit brain," Journal of Alzheimer's Disease, vol. 4, no. 6, pp. 523529, 2002.

[4] D. L. Sparks and B. G. Schreurs, "Trace amounts of copper in water induce $\beta$-amyloid plaques and learning deficits in a rabbit model of Alzheimer's disease," Proceedings of the National Academy of Sciences of the United States of America, vol. 100, no. 19, pp. 11065-11069, 2003.

[5] D. L. Sparks, "The early and ongoing experience with the cholesterol-fed rabbit as a model of Alzheimer's disease: the old, the new and the pilot," Journal of Alzheimer's Disease, vol. 15, no. 4, pp. 641-656, 2008.

[6] R. Deane, Z. Wu, A. Sagare et al., "LRP/amyloid $\beta$-peptide interaction mediates differential brain efflux of $A \beta$ isoforms," Neuron, vol. 43, no. 3, pp. 333-344, 2004.

[7] M. C. Morris, D. A. Evans, C. C. Tangney et al., "Dietary copper and high saturated and trans fat intakes associated with cognitive decline," Archives of Neurology, vol. 63, no. 8, pp. 1085-1088, 2006.

[8] D. J. Waggoner, T. B. Bartnikas, and J. D. Gitlin, "The role of copper in neurodegenerative disease," Neurobiology of Disease, vol. 6, no. 4, pp. 221-230, 1999.

[9] R. Squitti, D. Lupoi, P. Pasqualetti et al., "Elevation of serum copper levels in Alzheimer's disease," Neurology, vol. 59, no. 8, pp. 1153-1161, 2002.

[10] R. Ozcankaya and N. Delibas, "Malondialdehyde, superoxide dismutase, melatonin, iron, copper, and zinc blood concentrations in patients with Alzheimer disease: cross-sectional study," Croatian Medical Journal, vol. 43, no. 1, pp. 28-32, 2002.

[11] R. Squitti, P. Pasqualetti, E. Cassetta et al., "Elevation of serum copper levels discriminates Alzheimer's disease from vascular dementia," Neurology, vol. 60, no. 12, pp. 2013-2014, 2003.

[12] D. Strausak, J. F. B. Mercer, H. H. Dieter, W. Stremmel, and G. Multhaup, "Copper in disorders with neurological symptoms: Alzheimer's, Menkes, and Wilson diseases," Brain Research Bulletin, vol. 55, no. 2, pp. 175-185, 2001.

[13] S. Bucossi, M. Ventriglia, V. Panetta et al., "Copper in Alzheimer's disease: a meta-analysis of serum, plasma, and cerebrospinal fluid studies," Journal of Alzheimer's Disease, vol. 24, no. 1, pp. 175-185, 2011.

[14] J. Snaedal, J. Kristinsson, S. Gunnarsdóttir, Á. Ólafsdóttir, M. Baldvinsson, and T. Jóhannesson, "Copper, ceruloplasmin and superoxide dismutase in patients with Alzheimer's disease. A case-control study," Dementia and Geriatric Cognitive Disorders, vol. 9, no. 5, pp. 239-242, 1998.

[15] R. Squitti, P. Pasqualetti, G. Dal Forno et al., "Excess of serum copper not related to ceruloplasmin in Alzheimer disease," Neurology, vol. 64, no. 6, pp. 1040-1046, 2005.

[16] R. Squitti, G. Barbati, L. Rossi et al., "Excess of nonceruloplasmin serum copper in AD correlates with MMSE, CSF $\beta$ amyloid, and h-tau," Neurology, vol. 67, no. 1, pp. 76-82, 2006.

[17] Y. Gu, F. Oyama, and Y. Ihara, " $\tau$ is widely expressed in rat tissues," Journal of Neurochemistry, vol. 67, no. 3, pp. 12351244, 1996.

[18] M. Mercken, M. Vandermeeren, U. Lubke et al., "Monoclonal antibodies with selective specificity for Alzheimer tau are directed against phosphatase-sensitive epitopes," Acta Neuropathologica, vol. 84, no. 3, pp. 265-272, 1992.
[19] D. Strozyk, L. J. Launer, P. A. Adlard et al., "Zinc and copper modulate Alzheimer A $\beta$ levels in human cerebrospinal fluid," Neurobiology of Aging, vol. 30, no. 7, pp. 1069-1077, 2009.

[20] L. M. Sayre, G. Perry, P. L. R. Harris, Y. Liu, K. A. Schubert, and M. A. Smith, "In situ oxidative catalysis by neurofibrillary tangles and senile plaques in Alzheimer's disease: a central role for bound transition metals," Journal of Neurochemistry, vol. 74, no. 1, pp. 270-279, 2000.

[21] Q. F. Ma, Y. M. Li, J. T. Du et al., "Binding of copper (II) ion to an Alzheimer's tau peptide as revealed by MALDI-TOF MS, CD, and NMR," Biopolymers, vol. 79, no. 2, pp. 74-85, 2005.

[22] Q. Ma, Y. Li, J. Du et al., "Copper binding properties of a tau peptide associated with Alzheimer's disease studied by CD, NMR, and MALDI-TOF MS," Peptides, vol. 27, no. 4, pp. 841849, 2006.

[23] O. Ghribi, B. Larsen, M. Schrag, and M. M. Herman, "High cholesterol content in neurons increases BACE, $\beta$-amyloid, and phosphorylated tau levels in rabbit hippocampus," Experimental Neurology, vol. 200, no. 2, pp. 460-467, 2006.

[24] C. Ullrich, M. Pirchl, and C. Humpel, "Hypercholesterolemia in rats impairs the cholinergic system and leads to memory deficits," Molecular and Cellular Neuroscience, vol. 45, no. 4, pp. 408-417, 2010.

[25] O. Ghribi, M. Y. Golovko, B. Larsen, M. Schrag, and E. J. Murphy, "Deposition of iron and $\beta$-amyloid plaques is associated with cortical cellular damage in rabbits fed with long-term cholesterol-enriched diets," Journal of Neurochemistry, vol. 99, no. 2, pp. 438-449, 2006.

[26] A. L. Guillozet, S. Weintraub, D. C. Mash, and M. Marsel Mesulam, "Neurofibrillary tangles, amyloid, and memory in aging and mild cognitive impairment," Archives of Neurology, vol. 60, no. 5, pp. 729-736, 2003.

[27] E. Kok, S. Haikonen, T. Luoto et al., "Apolipoprotein Edependent accumulation of alzheimer disease-related lesions begins in middle age," Annals of Neurology, vol. 65, no. 6, pp. 650-657, 2009.

[28] L. A. Hansen, E. Masliah, D. Galasko, and R. D. Terry, "Plaqueonly Alzheimer disease in usually the Lewy body variant, and vice versa," Journal of Neuropathology and Experimental Neurology, vol. 52, no. 6, pp. 648-654, 1993.

[29] M. Yamada, "Senile dementia of the neurofibrillary tangle type (tangle-only dementia): neuropathological criteria and clinical guidelines for diagnosis," Neuropathology, vol. 23, no. 4, pp. 311-317, 2003.

[30] D. L. Sparks, "Cholesterol metabolism and brain amyloidosis: evidence for a role of copper in the clearance of $A \beta$ through the liver," Current Alzheimer Research, vol. 4, no. 2, pp. 165$169,2007$. 


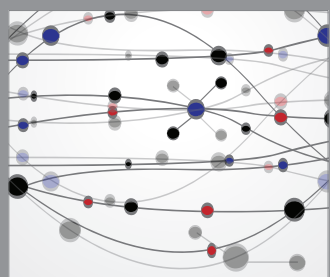

The Scientific World Journal
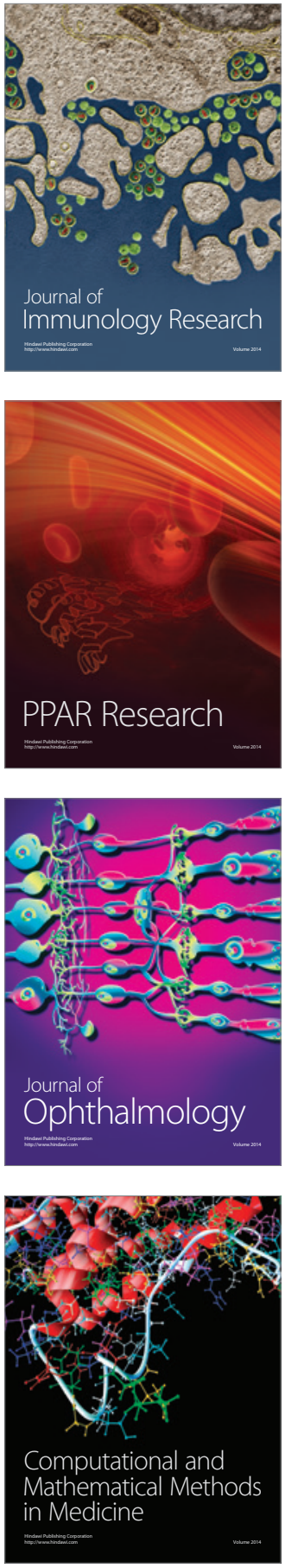

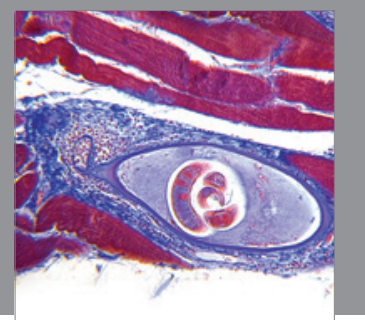

Gastroenterology

Research and Practice
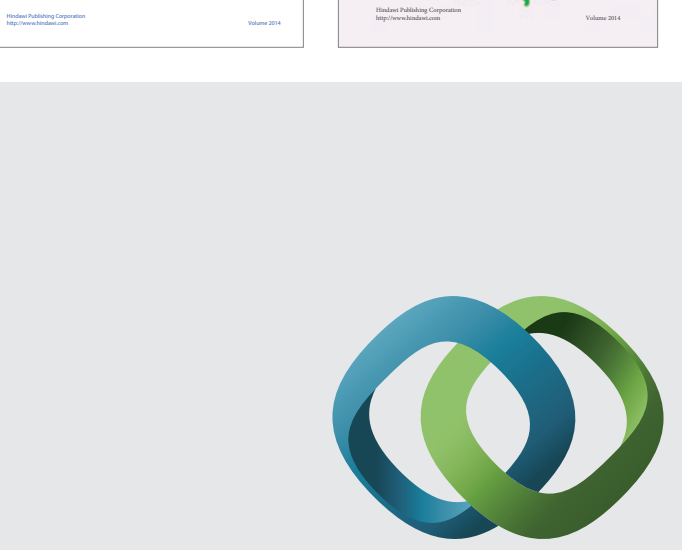

\section{Hindawi}

Submit your manuscripts at

http://www.hindawi.com
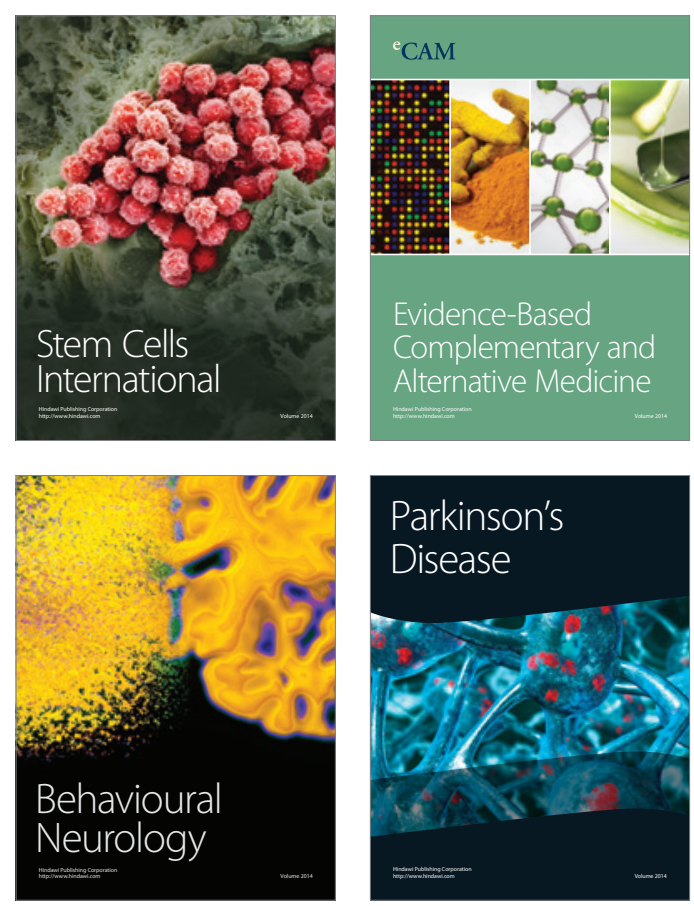

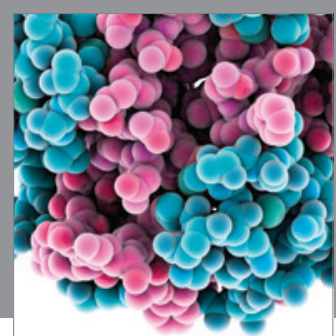

Journal of
Diabetes Research

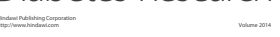

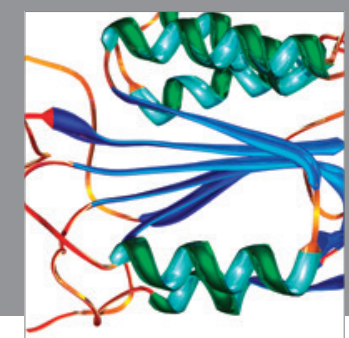

Disease Markers
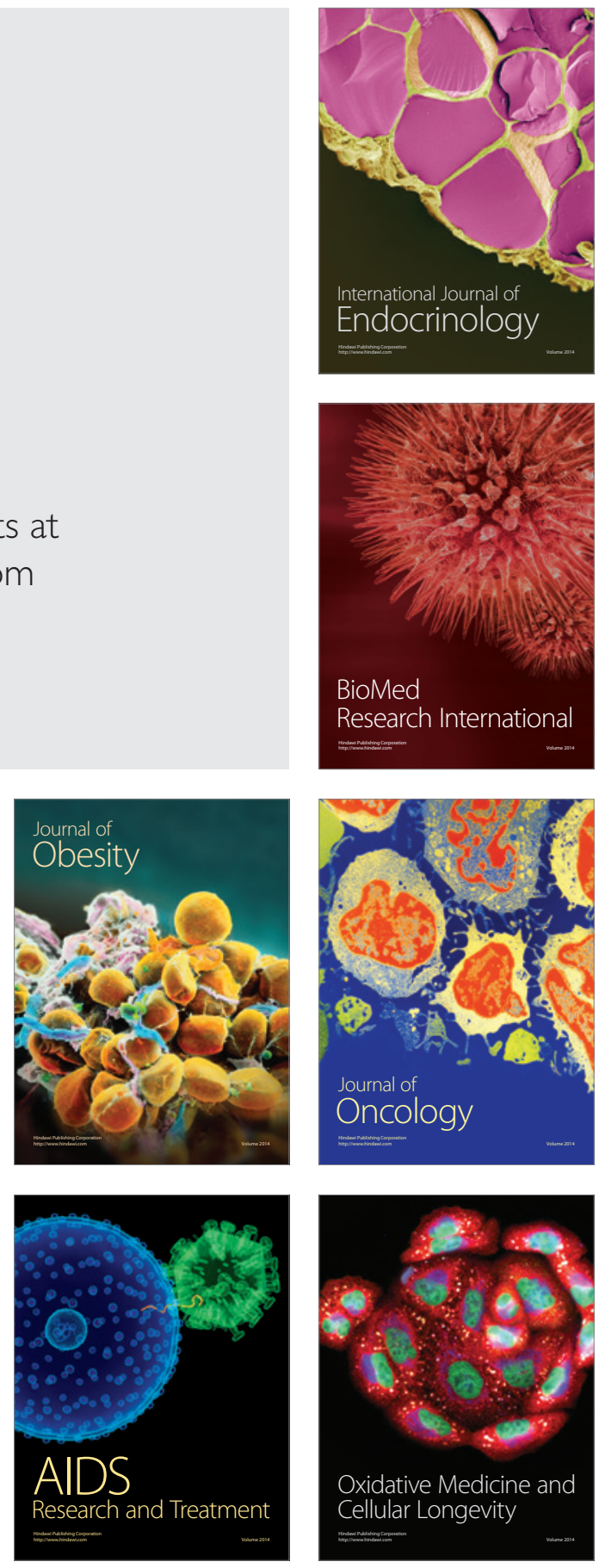